\title{
Observatoire international de géopolitique
}

Un projet de la Chaire Raoul-Dandurand en études stratégiques et diplomatiques

et du département de géographie de I'UQÀM

\section{PROGRAMME PRÉLI MI NAI RE \\ Colloque \\ « Ressources naturelles et conflits contemporains : nouveaux enjeux, nouveaux défis »}

29-30 septembre, 2005

200, rue Sherbrooke Ouest

Information : (514) 987-6781

chaire.start@uqam.ca

\section{LOUR 1}

\section{Accueil et inscription: $8 \mathrm{~h} 00-8 \mathrm{~h} 30$}

Mot de bienvenue: 8h30 - 8h45

- Michel J ébrak, vice-recteur à la recherche et la création, Université du Québec à Montréal.

\section{Conférence d'ouverture: 8h45 - 9h15}

- Représentant du Ministère Ressources naturelles Canada. À confirmer

\section{Panel 1: 9h15 - $10 h 45$}

Les impacts des ressources naturelles dans les rapports de force contemporains

- Oil, Geopolitics and War.

Michael T. Klare, Professeur, Department of Peace and World Security Studies, Five College.

- Dégradation de l'environnement et conflits régionaux.

Jacques Sironneau, Chef du Bureau des Affaires Juridiques à la Direction de l'Eau, Ministère de l'écologie et du développement durable de la France.

- Resources Governance and Armed Conflicts.

Philippe Le Billon, Professeur adjoint, Département de géographie, University of British Columbia.

- L'industrie minière dans les conflits armés: acteur, témoin ou otage?

Jean-Marc Lulin, Président, Azimut Exploration Inc. 


\section{Pause café: 10h45 - 11h00}

\section{Panel 2: 11h00 - 12h30}

L'Eurasie: la ruée vers l'or noir

- The Evolution of the Relationship between the Russian State and Big Oil. Fergal O'Reilly, Analyste, Bureau de l'évaluation internationale, Bureau du Conseil privé, Gouvernement du Canada.

- Insecurity and Instability in the Caspian Sea Region.

Robert Ebel, Directeur, Energy Program, Center for Strategic and International Studies.

- Oil and Gas and the Law in Russia - Systemic, Particular and Conceptual Problems.

Janet Keeping, Directrice, Russia Programmes, Canadian Institute of Resources Law, University of Calgary.

- Competition between China and Japan for Russia's Far East Oil Pipeline Project. Richard Giragosian, Chercheur associé, Institute for the Analysis of Global Security.

\section{Buffet: 12h30 - 14h30}

\section{Panel 3: 14h30 - 15H45}

\section{Panel Étudiant I}

- Environmental Change and Social Change in Ayacucho, Peru.

Tom Deligiannis, Candidat au doctorat, Département de science politique, University of Toronto.

- Opportunity and Willingness: A New Look at Natural Resources and Conflict. Kristine St-Pierre, Candidate à la maîtrise, Norman Paterson School of International Affairs, Carleton University.

- Conflits autour des hydrocarbures au Nigeria : enjeux de pouvoir et interdépendances.

Philippe Sébille-Lopez: Candidat au doctorat, Département de science politique, Institut français de Géopolitique, Université Paris 8.

- 2001 National Energy Policy : La guerre pour le pétrole, une nouvelle facette de la lutte américaine en Colombie.

Jorges Andres Rave, Candidat à la maîtrise, Département de science politique, Université du Québec à Montréal.

\section{Pause café: 15h45 - 16h00}




\section{Panel 4: 16h00 - 17h30}

L’Asie: la face cachée de la croissance économique

- Water Resources Management in China: from Conflicts to Contracts and Back Again?

Alana Boland, Professeure adjointe, Département de géographie, University of Toronto.

- Energy Conflict in the Asia-Pacific.

James J. Barnes, Bonner Means Baker Research Fellow, James A. Baker III Institute for Public Policy. À confirmer

- Les investissements directs étrangers dans le secteur des ressources naturelles en Chine: vers une libéralisation?

Mark Worrall, Économiste - Asie du Nord, Exportation et Développement Canada.

- Le rôle géopolitique de la ressource forestière en Asie du Sud-Est. Cas de I'Indonésie et du Loas.

Yann Roche, Professeur adjoint, Département de géographie, Université du Québec à Montréal.

\section{LOUR 2}

\section{Accueil et inscription: $8 \mathrm{~h} 30$ - $9 \mathrm{~h} 00$}

\section{Panel 5: 9h00 - 10h30}

L'Afrique et le Moyen-Orient: une instabilité chronique?

- Corporate Warriors in the Congo

Jim Freedman, Professeur émérite, Département d'anthropologie, University of Western Ontario.

- Water Scarcity, Oil Abundance: Resources and Conflict in the Middle East and North Africa.

Miriam R. Lowi, Professeure titulaire, Département de science politique, The College of New Jersey.

- Assessing the Oil Factor in the New US Middle East Policy.

Pierre Noël, Analyste, Institut français de relations internationales.

- Petroleum, Poverty and Security in Africa

Keith J. Myers, Chercheur associé, Africa Programme, Chatham House.

Pause café: 10h30 - 10h45 


\section{Panel 6: 10h45 - 12h00}

\section{Panel Étudiant II}

- Échelles géographiques et rôle des ressources naturelles dans les conflits : une approche comparative.

Julien Vandeburie, Candidat au doctorat, Département de géographie, Université Libre de Bruxelles.

- Water Scarcity and Potential for Conflict in South Asia.

Anita Singh, Candidate à la maîtrise, Département de science politique, University of Calgary.

- Nouveaux rapports de force et conflits miniers: enjeux et impasses du cas péruvien.

Myriam Laforce, Candidate à la maîtrise, Département de science politique, Université du Québec à Montréal; Chercheure, Groupe de recherche sur les activités minières en Afrique.

\section{Buffet: 12h00 - 14h00}

\section{Panel 7: 14h00 - 15h30}

L’Amérique du Sud: les soulèvements populaires

- Venezuelan Oil in Hostage?

Sandy Tolan, Professeur, Département de journalisme, UC Berkeley. À confirmer

- Resource Governance and Popular Protest in Bolivia: The Cases of Water and Gas.

Tom Perreault, Professeur adjoint, Département de géographie, Maxwell School of Syracuse University.

- Forest management and conflict in Latin America

Ronnie de Camino, Professeur, Département des Ressources naturelles et de la Paix, University for Peace. À confirmer

- Ressources naturelles, dégradation environnementale, terrorisme écologique et conflits en Colombie et dans ses zones de frontières.

Stéphanie Lavaux, Professeure, Faculté de science politique et de relations internationales, Université du Rosario.

\section{Pause café: 15h30 - 15h45}




\section{Panel 8: 15h45 - 17h15}

L'Amérique du Nord et l'Europe : au-delà de la consommation

- Les projets de transfert d'eau du Canada vers les États-Unis : un enjeu politique réel pour le Canada.

Frédéric Lasserre, Professeur, Département de géographie, Université Laval;

Directeur, Observatoire de recherches internationales sur l'eau.

- Les ressources naturelles et les règlements judicaires des différends territoriaux en Amérique du Nord et en Europe.

George Labrecque, Professeur, Faculté de sciences politiques et économiques, Collège militaire royal du Canada.

- Environmental Issues within the Energy Sectors.

Douglas Bruchet, Vice-president senior, Energy \& Environment Research.

Team, Canadian Energy Research Institute.

- La gestion de l'eau à l'épreuve du partenariat public privé : l'exemple de Grenoble.

Élisabeth Vallet, Chercheure, Chaire Raoul-Dandurand en études stratégiques et diplomatique, Université du Québec à Montréal.

Mot de la fin: 17h15-17h30 